\title{
Study on positioning precision for moving vehicle with different tag-sensor arrangement patterns
}

\author{
Weidong Ao ${ }^{1, a}$, Enzhan zhang ${ }^{2, b}$, Jishen Liang ${ }^{3, c}$ and Dongxuezhang ${ }^{4}$ \\ ${ }^{1}$ Chongqing Vocational college of Transportation, Chongqing, 402247, China \\ ${ }^{2}$ Lanzhou University of Technology, Lanzhou, 730050, China \\ ${ }^{3}$ Communication NCO Academy,Army Engineering Uuniversity of PLA, Chongqing, 400035, China \\ ${ }^{4}$ Chongqing Huiling New Technology Com Ltd, Chongqing, 401336, China

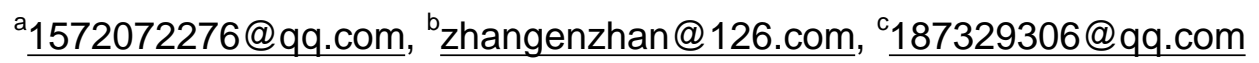

Keywords: vehicle positioning, position precision, arrangement patterns

Abstract. The Radio Frequency IDentification (RFID) based Active Vehicle Positioning (RAVP) is a feasible and high precision positioning method for moving vehicle on road when someplace uncovered by the signal of GPS or some other satellite based positioning systems. In this paper, RAVP is introduced and the precision of it is analyzed in different tag arrangement patterns through Monte Carlo simulation. The results show that the triangular tag arrangement has a reasonable position precision (0.6meter) which has high precise to locate vehicles on their belonging lanes, and hence this method can be used for lane departure warning and autonomous driving system.

\section{Introduction}

Nowadays, vehicle positioning is in great demand for many safety applications, even though various kinds of positioning systems have been proposed. Global Positioning System (GPS) is available for vehicle positioning and navigation [1,2]. Mobile network based positioning [3], which has lower precision about several kilometers, is being used for location-based services (LBS), such as location aware electronic shopping, ordering and advertising. Moreover, the vision-based positioning [4]-[7], could be classified into two parts: one is to detect road features (lane markings and boundaries), and to determine the position of the vehicle with respect to the lane. The other one estimates the vehicle position by collecting signpost marks, such as milestone and direction marks on the road. However, both these two categories could only provide relative position.

The previous positioning methods could not locate vehicles onto their belonging lanes with global position coordination. Paper $[8,9,10]$ have shown that many RFID-based positioning related works mainly focus on indoor positioning. In paper [11], the author firstly introduced the idea of using RFID technology for vehicle positioning to improve the accuracy of a navigation system on the road. The preliminary results on RFID communication speed and tag database access time proved that reading location information from tags is feasible for moving vehicles. In paper [12], more factors have been taken into consideration to realize RFID based Active Vehicle Positioning (RAVP), such as installation position of the RFID reader and tag, incline angle and main lobe width of both RFID reader and tag. The most important contribution of these papers is that RAVP is a feasible and useful positioning method for road traffic, which can provide higher precision when the GPS and some other satellite based positioning systems outage.

Nevertheless, paper [11, 12] did not analyze the positioning precision for different matrix patterns of tag arrangement. In this paper, we present analysis on the precision of RAVP in different tag matrix patterns. Although, in [13], the author has presented some valuable results of choosing tag patterns, but the situation being taken into account is in room with a random moving robot, which is quite different from the road traffic scenario, our research is based on the vehicles which are moving along a pre-set road at a high speed. In the following, we will see that, when the tag coverage overlaps, the performance can be improved especially in the overlapped regions. Therefore, in this paper, we mainly focus on the overlapped cases for both triangular and square tag patterns and present Monte Carlo simulation results. The results prove that the positioning precision of triangular pattern is higher 
than square pattern in the same tag density. To simply our paper, the standards (Operating frequency, radio propagation, etc.) of both RFID tag and reader would not be restated in this paper, for these standards and performance in a harsh environment is well elaborated in [14].

The rest of this paper is organized as follows. We give a brief overview of RAVP scheme in section I. Tag arrangement patterns are given in section II. The average positioning precision (APP) is analyzed in section III. Simulation results are shown in section IV. Finally, conclusion is in section V.

\section{RFID based active vehicle positioning method}

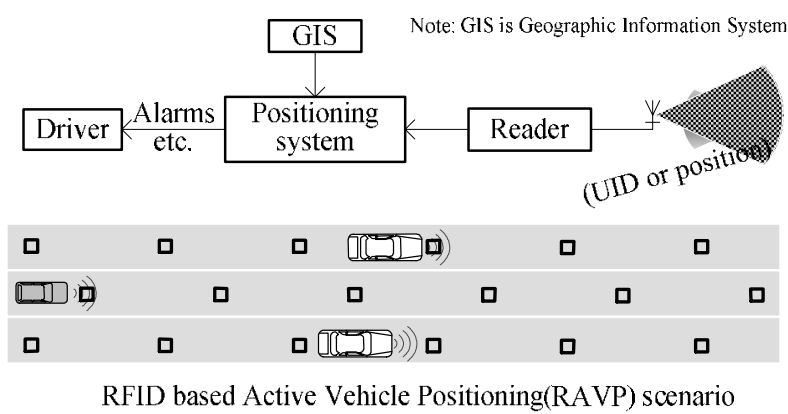

Fig. 1 RAVP system model

The RAVP system model is given in Fig. 1. Vehicle equipped with RFID reader passes over the road covered with a set of tags. In this model, RAVP is a multi-tag based positioning technique. The RAVP system is composed of at least four parts: RFID tags, reader, computation facilities and Geographic Information System (GIS). Here, RFID tags are installed on the road. Reader is mounted on the vehicle that can trigger multiple tags around. Computation facilities calculate vehicle's position based on location information of scanned tags. GIS supports vehicle locating and navigating.

As the vehicle moves along the road, the RFID reader continuously generates radio frequency carrier waves to activate tags in its reading range(In this paper, the radiated radius of both reader and tag are called reading range, but the length of them are different, which depends on the radiated energy from reader and tag). Certain tags will wake up when they absorb enough power from the radiated waves. The awakened tag/tags then reflect a portion of the energy absorbed from the reader radiation back to the reader. The reflected energy is modulated by the data stream from the tag's chip. The variation of the reflected radio wave will be picked up by the reader and be demodulated, thus, position information stored in the RFID tags can be retrieved. The position information in tag can be acquired by using differential-GPS or some other methods.

The performance of RAVP is heavily dependent on how densely tags are distributed along the road and how they are arranged. However, the increased tag distribution density may cause excessive tag installation cost as well as the technical problem of duplicated tag readings. Therefore, tag distribution density and tag arrangement pattern should be carefully designed for reasonable position precision as well as to minimize overall investment and improve positioning accuracy, which is the focus of this paper. Otherwise, the installation work is a huge government project, which can be done by Highway Department or Ministry of Transport. Tags are made into nail shape embedded into hard device in order to install conveniently on to the road surface, and the damage to the road can be decreased.

\section{Tag arrangement pattern}

The most important thing in tag arrangement is that the pattern should cover the road surface uniformly, this condition will be satisfied easily if the arrangement pattern that are used have the same shape and size. For shapes to fill the plane without gaps or overlaps, their angles, when arranged around a point, must have measures that add up to exactly $360^{\circ}[15]$. Therefore, the only regular polygons that create uniform arrangement patterns are equilateral triangles, squares, and regular 
hexagons as shown in Fig. 2. As can be seen in this figure, the triangular pattern has a better coverage than hexagonal pattern, thus only the triangular and square cases will be discussed in this paper.

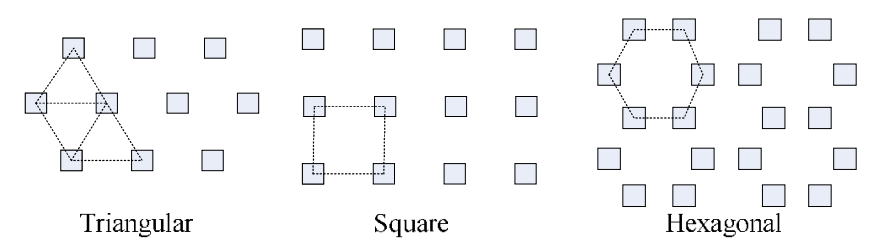

Fig. 2 Tag arrangements

For triangular and square tag arrangement patterns, there are different area partitions even for the same arrangement pattern when the ratio of tag radius $(R$, tag reading range) to tag distance $(L)$ varies, which will be discussed below.

\section{Triangle arrangement scheme}

For triangle arrangement, we can find different shapes for different ratios of $\mathrm{R}$ to $\mathrm{L}$ in Fig. 3. $\mathrm{L}$ is fixed. When $\mathrm{R}$ increases, we can observe that there are at least four different cases, (a) $R \leq \frac{1}{2} L$, (b) $\frac{1}{2} L<R \leq \frac{1}{\sqrt{3}} L$, (c) $\frac{1}{\sqrt{3}} L<R \leq \frac{\sqrt{3}}{2} L$, (d) $R>\frac{\sqrt{3}}{2} L$.

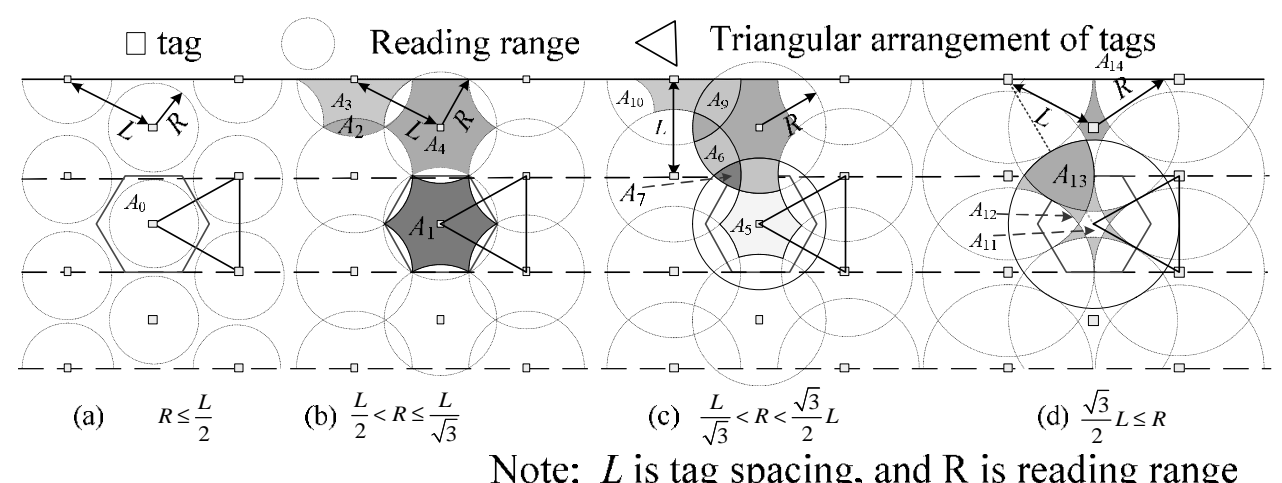

Fig. 3 Triangular arrangement pattern

In Fig. 3(a), there are some areas where the reader cannot sense the tags. In this paper we call this area "dead zone". For convenient analyzing, assuming tag distance $L$ is fixed, if the radius $R$ increases, the number and shape of the defined area will not change but only in terms of size when $R$ falls into those regions.

There exist four area types in Fig. 3. For example, as shown in Fig. 3(b) when $L / 2<R \leq L / \sqrt{3}$, two of which are in the middle of the lane, A1, A2. A1 defines an area covered by one tag while outside of other all tags' coverage, while A2 defines an area covered by two adjacent tags while outside that of all other tags. A7 defines areas covered by three tags in Fig. 3(c). In Fig. 3(d), the area is A13. There are more than four areas exist which will result in some shapes are too complex and useless for real scenario, difficult computing of the location. This case will not be analyzed in this paper. 


\section{Square arrangement scheme}

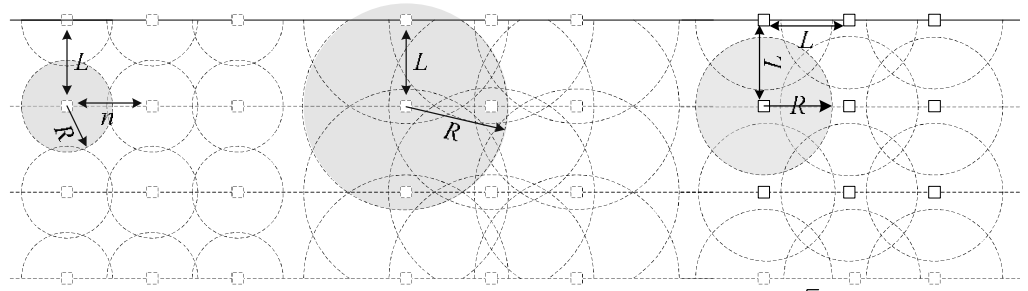

(a) $R<\frac{\sqrt{2}}{2} L$

(b) $R>L$

(c) $\frac{\sqrt{2}}{2} L \leq R \leq L$

Note: $L$ is tag spacing, and $R$ is reading range

Fig. 4 Square arrangement pattern

For square case, there are also "dead zones", when $R<\frac{\sqrt{2}}{2} L$ as shown in Fig. 4(a). In Fig. 4(b), when $R>L$, there will be some area, where the reader can sense more than 5 tags at the same time. This is same to triangular case in Fig. 3(d). To save space, we just present the APP calculation formula on the square tag arrangement in section-IV when $\frac{\sqrt{2}}{2} L \leq R \leq L$ as shown in Fig. 4(c).

\section{Average positioning precision calculation}

Assuming that the reader's antenna is unidirectional, we focus on APP of all areas on the road in our evaluation by simulations. For each area, say $A_{i}$, its APP can be written as:

$$
A P P=\frac{\iint \sqrt{\left(x-x_{0}\right)^{2}+\left(y-y_{0}\right)^{2}} d x d y}{\iint d x d y}
$$

Where $(\mathrm{x}, \mathrm{y})$ is the coordinates of the reader - the vehicle's position, and $\left(\mathrm{x}_{0}, \mathrm{y}_{0}\right)$ is the location point of the vehicle's belonging area.

For $n$ vehicles, it can be rewritten as:

$$
A P P=\left\{\begin{array}{cc}
\frac{1}{n} \sum_{i=1}^{n} A P P_{i}, & A P P_{i} @ \mid p_{i}-z_{k} \|, p_{i} \in A_{k} \\
n o t \text { valid } & p_{i} \notin A_{k} \text { for all areas }
\end{array}\right.
$$

Where $\| \mathrm{d}$ is 2-norm of $p_{i}$ and $z_{k}, p_{i}$ is the position of $i^{\text {th }}$ vehicle, and $z_{k}$ is the center of the $i^{\text {th }}$ vehicle's belonging area $A_{k}$ which is given by:

$$
\begin{aligned}
& z_{k} @\left(x_{k}, y_{k}\right)=\operatorname{mean}\left\{\left(x_{j}^{k}, y_{j}^{k}\right)\right\}, \quad\left(x_{j}^{k}, y_{j}^{k}\right) \in A_{k} \\
& x_{k} @ \frac{1}{n_{k}} \sum_{j=1}^{n_{k}} x_{j}^{k}, \quad y_{k} @ \frac{1}{n_{k}} \sum_{j=1}^{n_{k}} y_{j}^{k}
\end{aligned}
$$

where $n_{k}$ is total number of vehicles fall into area $A_{k}$. The index of areas, $k=[1, K], K$ is the total number of areas which is different for triangular and square patterns.

\section{Simulation}

In above analysis, the vehicle location is assumed evenly random distributing on the road. However, it is not the case in real traffic. Therefore, both "pure" random (uniform distribution) case and real traffic (Pseudo-Gaussian distribution, which will be given in the follow subsection A.) case are considered. 


\section{Real Traffic Scenario}

Real traffic data of cars passing each section of the partition was recorded by taking a 30minute video of both directions of traffic flow, as shown in Fig. 5. As illustrated in Fig. 5, each of the three lanes is divided into nine partitions, which is precise enough for calculating vehicle distribution.

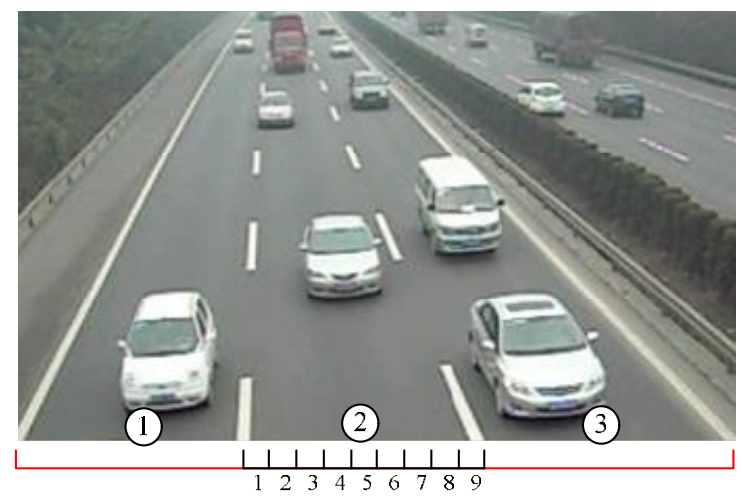

Fig. 5 Real traffic scenario

The number of vehicles passing each partition in the recorded time is shown in Table 1 and Table 2 from the table a graph of vehicle distribution is plotted in Fig. 6. It can be observed from Fig. 6 that the vehicles are pseudo-Gaussian but not uniformly distributed across the lane.

Table 1 Number of vehicles in each partition (Direction-1)

\begin{tabular}{|l|l|l|l|l|r|r|r|r|l|l|}
\hline $\begin{array}{c}\text { Partition } \\
\text { s }\end{array}$ & 1 & 2 & 3 & 4 & 5 & 6 & 7 & 8 & 9 & $\%$ \\
\hline Lane-1 & 0 & 4 & 1 & 3 & 47 & 2 & 1 & 6 & 0 & $\begin{array}{l}19.06 \\
\%\end{array}$ \\
\hline Lane-2 & 0 & 9 & 1 & 5 & 10 & 5 & 2 & 2 & 0 & $\begin{array}{l}35.22 \\
\%\end{array}$ \\
\hline Lane-3 & 6 & 1 & 2 & 6 & 11 & 8 & 2 & 1 & 0 & $\begin{array}{l}45.72 \\
\end{array}$ \\
\hline
\end{tabular}

Table 2 Number of Vehicles in each partition (Direction-2)

\begin{tabular}{|c|c|c|c|c|c|c|c|c|c|c|}
\hline $\begin{array}{c}\text { Partition } \\
\text { s }\end{array}$ & 1 & 2 & 3 & 4 & 5 & 6 & 7 & 8 & 9 & $\%$ \\
\hline Lane-1 & 0 & 5 & 1 & 2 & 5 & 3 & 2 & 1 & 3 & $\begin{array}{c}24.21 \\
\%\end{array}$ \\
\hline Lane-2 & 0 & 3 & 1 & 4 & 8 & 5 & 1 & 4 & 0 & $\begin{array}{c}32.09 \\
\%\end{array}$ \\
\hline Lane-3 & 0 & 8 & 2 & 6 & 9 & 7 & 3 & 9 & 0 & $\begin{array}{c}43.70 \\
\%\end{array}$ \\
\hline
\end{tabular}
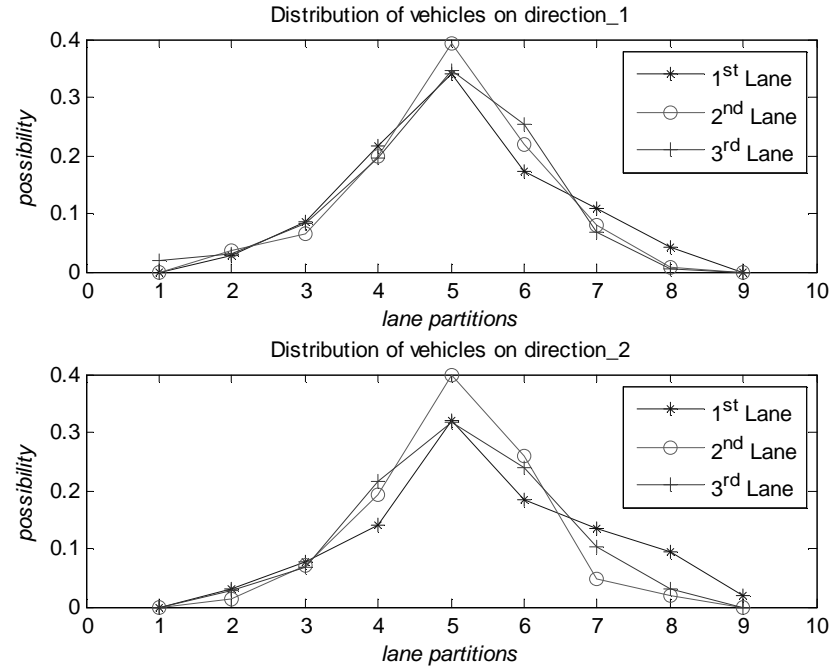

Fig. 6 Vehicles distribution on different lanes

\section{Simulation results}


For a given tag distribution density, the tag arrangement pattern over the road affects the performance of RAVP. We will compare the APP of triangular arrangement and square arrangement with the same density in Uniform and Gaussian distribution cases through Monte-Carlo simulation.

The simulation settings and the tag arrangement scenario are shown in Table 3 and Fig. 7.

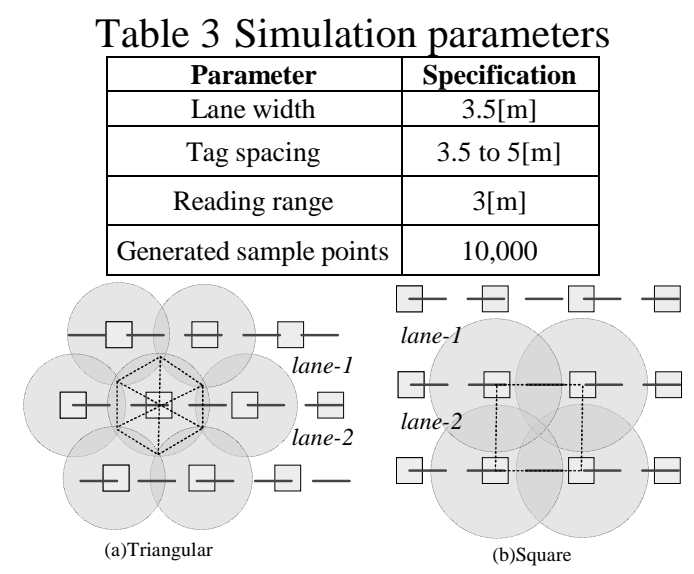

Fig. 7 Triangular and square tag arrangement scenario for simulation

The average positioning precision of the two arrangement patterns is illustrated in Fig. 8, where the density $\mathrm{D}$ relates to the tag spacing $\mathrm{L}$ for square and triangular patterns respectively as follows:

$$
L_{\text {square }}=1 / \sqrt{D}, \quad L_{\text {Triangular }}=\sqrt{2 /(\sqrt{3} D)}
$$

Next, we compare the APP of triangular and square tag pattern in the same tag density.

Fig. 8 demonstrates the Monte-Carlo simulation results of the two arrangement patterns in both uniform and Gaussian distributions.

When reading range $\mathrm{R}=3$ meter is fixed, following $\frac{\sqrt{3}}{3} L \leq R \leq \frac{\sqrt{3}}{2} L$ and equation (4), we can obtain $2 \sqrt{3} \leq L \leq 3 \sqrt{3}$ and $\frac{2}{27 \sqrt{3}} \leq D \leq \frac{1}{6 \sqrt{3}}$ (about $0.0427 \leq D \leq 0.0962$ ) in triangular arrangement scheme. And following $\frac{\sqrt{2}}{2} L \leq R \leq L$ and equation (4), we can obtain $3 \leq L \leq 3 \sqrt{2}$ and $1 / 18 \leq D \leq 1 / 9$ (about $0.0556 \leq D \leq 0.1111$ ) in square arrangement scheme. The region of overlapping $\mathrm{D}$ is $\frac{1}{18} \leq D_{\text {overlap }} \leq \frac{1}{6 \sqrt{3}}$ (about $0.0556 \leq D \leq 0.0962$ ) of triangular and square arrangement scheme. In this region, as can be seen in Fig. 8, the APP of triangular and square arrangement are almost same, the maximum difference is more than 0.38 meter in Uniform distribution case of vehicle location. In Gaussian distribution case, the APP of triangular arrangement is obviously higher than square arrangement. The maximum difference is more than 0.5 meter. So we can conclude that triangular arrangement can provide a higher positioning precision under the same condition in real traffic scenario $(\mathrm{R}=3 \mathrm{~m}, \mathrm{~L}=3.5 \sim 5 \mathrm{~m}$, vehicles across the lane take pseudo-Gaussian distribution).

If we try to reduce tags cost, we need to decrease $\mathrm{D}$ as much as possible. If $\mathrm{D}$ decreases, the tag spacing increases and the precision of positioning decreases. In practical deployment, the average length of the vehicles and the width of the lane should been taken into account in determining the tag spacing. The average length of the vehicles are usually 4 5meters and the width of the lane is 3.5 meter, consequently we can choose the tag spacing 3.5meters which equal to lane width. And it satisfies $\frac{\sqrt{3}}{3} L \leq R \leq \frac{\sqrt{3}}{2} L$ and $\frac{\sqrt{2}}{2} L \leq R \leq L$ in triangular scenario and square scenario separately when the tag reading range $\mathrm{R}$ is 3 meter. Thus, the triangular arrangement pattern has a higher APP than square arrangement pattern in Gaussian-Distribution case as shown in Fig. 8. This means the triangular tag arrangement has a reasonable position precision $(0.6$ meter) and improves positioning accuracy for RFID based active vehicle positioning in real traffic scenario. 

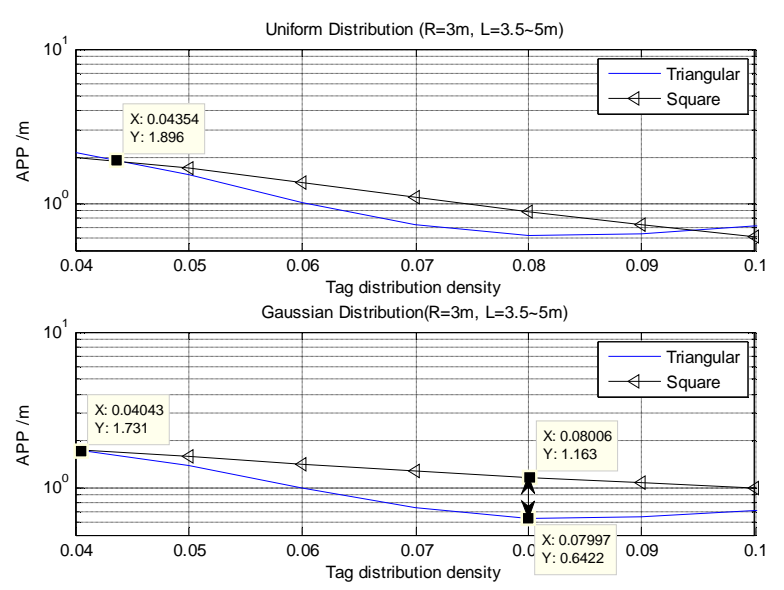

Fig. 8 APP versus tag distribution density

\section{Conclusion}

RAVP is an active, feasible and high precision positioning method for moving vehicles on road when the GPS and other satellite based positioning systems outage. This paper presented the precision analysis of RAVP with square and triangular tag arrangement patterns by using Monte Carlo simulation. The results demonstrated clearly that the average positioning precision in triangular pattern is higher than that in square pattern in practical deployment of tags. Thus, in most cases triangular tag arrangement shall be preferred over square tag arrangement to improve positioning precision for RAVP. In addition, the performance of RAVP is precise enough to locate vehicles on their belonging lanes. It is useful for lane departure warning, vehicle collision warning and autonomous driving system.

\section{Acknowledgement}

This work was financially supported by the Science Foundation of Gansu Province (Grant: 1610RJZA25).

\section{References}

[1] V. Havyarimana, D. Hanyurwimfura, P. Nsengiyumva and Z. Xiao: A novel hybrid approach based-SRG model for vehicle position prediction in multi-GPS outage conditions, Information Fusion, Vol.41, p.1-8. (2018)

[2] S. Capkun, M. Hamdi and J. P. Hubaux: GPS-free positioning in mobile ad-hoc networks, Cluster Computing, Vol.5, No.2, p. 157-167. (2002)

[3] S. Sheng-li, X. Jian-min and Q. Zhong: Vehicle Positioning Based on CDMA and GSM Networks, Journal of South China University of Technology (Natural Science Edition). Vol.35, No.2, p.50-53. (2007)

[4] N. I. Kotov, S. B. Berkovich, R. N. Sadekov, A. V. Sholokhov, A. S. Lychagov and U. V. Likholay: Using vision systems to determine the vehicle position on the road, 2017 24th Saint Petersburg International Conference on Integrated Navigation Systems (ICINS), p.1-3. (2017)

[5] S. J. Mankar, M. Demde, P. Sharma: Design of computer vision intelligent system for lane detection, 2016 Online International Conference on Green Engineering and Technologies (IC-GET), p. 1 - 3. (2016)

[6] B. Rong-chang, H. Fen Tsai and Y. Chung-Ping: A fast and effective approach to lane marking and neighboring vehicles detections based on vision/GPS sensing together with 
Vehicle-to-Vehicle communication, 2009 Ninth International Conference on Hybrid Intelligent Systems, p. 3-8. (2009)

[7] H. Pei-Yung, Y. Chun-Wei, H. Shih-Shinh and F. Li-Chen: A protable vision-based real-time lane departure warning system: day and night, IEEE TRANSACTIONS ON VEHICULAR TECHNOLOGY, Vol. 58, No. 4, p. 2089-2094. (2009)

[8] S. Park and H. Lee: Self-Recognition of Vehicle Position Using UHF Passive RFID Tags, IEEE Transactions on Industrial Electronics, Vol. 60, No. 1, p. 226-234. (2013)

[9] M. Y. Ahmad and A. S. Mohan: Nover bridge-loop reader for positioning with HF RFID under sparse tag grid, IEEE Transactions on industrial electronics, Vol. 6, No. 1, p. 555-566. (2013)

[10] B. Olszewski, S. Fenton, B. Tworek, J. Liang and K. Yelamarthi: RFID positioning robot: An indoor navigation system, Electro/Information technology, 2013 IEEE international conference on, SD, USA, p. 9-11. (2013)

[11] H. D. Chon, S. Jun, H. Jung and S. W. An: Using RFID for accurate positioning, Journal of Global Positioning System Vol.3, No.1-2, p.32-39. (2004)

[12] Z. Enzhan, K. Yujun, J. Weili and U. M. Ahmed: Active RFID positioning of vehicles in road traffic, Communications and Information Technologies (ISCIT), 2011 11th International Symposium on, p.222-227. (2011)

[13] S. Kim: RFID tag arrangement for mobile robot localization, Sensors, 2009 IEEE, p.621-624, (2009)

[14] http//www.czgaote.com.

[15] http://www.redmond.k12.or.us/14552011718214563/lib/14552011718214563/Lesson_7.4.p df. 